\title{
Cultural Heritage Digitalization on Traditional Sundanese Music Instrument Using Augmented Reality with Multiple Marker Method
}

\author{
Budi Arifitama*), Ade Syahputra \\ Program Teknik Informatika Universitas Trilogi \\ Jl.TMP Kalibata no.1 Kampus Stekpi/Trilogi, Jakarta Selatan
}

\begin{abstract}
Research into cultural heritage which implements augmented reality technology is limited. Most recent research on cultural heritage are limited on storing data and information in the form of databases, this creates a disadvantage for people who wants to see and feel at the same moment on actual cultural heritage objects. This paper, proposes a solution which could merge the existing cultural object with people using augmented reality technology. This technology would preserve traditional instrument in the form of $3 D$ object which can be digitally protected. The result showed that the use of augmented reality on preserving cultural heritage would benefit people who try to protect their culture.
\end{abstract}

Keywords - Augmented reality; Digital preservation; Traditional music instrument; Cultural heritage

\section{INTRODUCTION}

Cultural heritage is one of the most important things for a nation, as it gives every nation a unique identity and different tradition for each nation. Indonesia is an archipelago based nation that has around 1340 ethnic living in a various region on a bigger island such as Sumatra, Java, Kalimantan, Sulawesi and Papua, which gives a huge amount of unique culture that needs to be protected and preserved. Every time a language or culture is lost, we lose an irreplaceable and exquisite way of being. Cultural preservation prioritizes the need to protect and restore all forms of cultural diversity, it recognizes the many strands of culture such as languages, arts, buildings, dances, folklore, crafts, sacred sites and much more.

Sundanese is one of the many ethnics in Indonesia, located in the western region of the Java island. Like every local ethnic, nowadays modern Sundanese still practice their tradition such as in the musical instruments. The lack of attention on preservation, made people tend to ignore its importance and abandon the practice of local tradition. Each time a tradition is torn down without a trace, or a gathering place paved over, a strand of culture is lost . At the moment, conventional preservation still has many flaws, it needs the presence of a group of people to practice their culture to remind them of their tradition. These problem needs to be solved by finding an appropriate solution which applies the use of technology.

*) Budi Arifitama

Email: budiarif@trilogi.ac.id
Technology happens to be the most important things in our life nowadays. Most people use technology to help manage their daily activities, we can see from the age of teenager till adult are using technology for work, education, and entertainment. The vast expansion and penetration of mobile devices made it easier for people to obtain devices which create an opportunity for a researcher to spread technological advancement for the people. The technology used in this research is based on augmented reality to imitate traditional Sundanese music instruments.

The characteristics of augmented reality systems can be further understood from three classical and widely used criteria [1]. The first view combines virtual world and real world. Augmented reality requires a display technology that allows the user to see virtual and real information in a combined view. The barrier between the two worlds seems to merge into one environment. The second view is using 3D perspective. Augmented Reality relies on an intimate coupling between the virtual and the real world that is based on their geometrical relationship. This makes it possible to render the virtual content with the correct placement and 3D object the same as the actual world. The last view is on real-time interaction. Augmented reality system must run at interactive frame rates, such that it can superimpose information in realtime and allow user interaction.

Augmented reality moves from the industrial approach to mass technology which can be used in our life [2], [3]. It can be defined as an innovation by emerging real world with enhancement of computer-generated content that is connected to particular places. In other words, augmented reality permits the digital content to be effortlessly superimposed and intermingled into our insights and conception of the real world [4].

Specht et al. [5] described an overview of augmented reality, the use of augmented reality in the various field of our daily life. The research done gives a wide knowledge on opportunities which uses augmented reality as a media. Yuen et al. [6] proposed a research based on preserving cultural heritage by applying the way of interaction between visitor in a museum, it also describes the urgency of digitalizing artifact in the museum, which categorized into four methods of the exhibition such as a premodern museum, modern museum, cyber museum and digital museum. The implementation form of augmented reality in a museum 
are information service and the explanation of artifact uses and function. Mayur et al. [7] proposed a guideline to be followed for developing software from the beginning phase of the cycle and until the end of the cycle, performing a simulation study for software development life cycles. It is composed of six such as requirement analysis, design analysis, implementation, testing, and maintenance. Each phase must be finished before conducting the next phase of the cycle. Kolstee and Eck [8] described the use of augmented reality in the advertisement area, it discussed the portability and easiness of using a mobile device which is a smart phone for augmented reality. It shows how the augmented reality is implemented on an advertisement of a restaurant which share product information, booking details, and 3D layout. Kim and Kim [9] described the use of augmented reality in educational learning purpose for meta-cognitive learning that composed of 4 cognitive system such as aggregation, enrichment, and synchronization framing. It also describes a game logic case study using the humancomputer interaction pattern on augmented reality. Azuma [10] proposed about augmented reality test cases on education, which resulted in several reactions between the student and the educator. Most of the student reacts positively on the use of augmented reality in their classroom while most educators feel alarmed as id augmented reality will "overtake" their classroom. Antonioli et al. [11] whom also implement augmented reality in a museum environment, implement the technology on artifact and painting. The paintings that are researched are van Gogh's painting. It proved that augmented reality is suitable for preserving cultural heritage.

In this research, four traditional Sundanese instruments will be developed using augmented reality multiple markers such as "angklung", "kendang", "suling" and "saron". The uses of multiple markers on augmented object gives alternative solution on how to capture augmented object on different markers at the same time. The research applied is based on finding interactive ways between users and augmented reality as preserve traditional instrument digitally.

\section{METHOD}

This research will use waterfall cycle as a framework to develop the research and is divided into six phases by following the waterfall cycle, each phase must be performed with caution to evade software development failure. The research starts from analysis requirement, analysis design, coding, testing, and support phase.

The first activity is to collect data and research information by interview and observation based on local community in the Western Java. The data and information gathered are traditional instruments history, instrument shapes and the sounds and will be used as primary information in making 3D instruments for the next phase of the research.

The second activity is 3D object design. This activity is done after analyzing data and information gathered before on traditional instrument which helps in specifying hardware and system requirements and also helps in defining overall system architecture. In this research, based on data gathered from the first phase, four shapes of instrument is designed in $3 \mathrm{D}$ to imitate the local instrument to preserved digitally.

The third activity is implementing the object designed with multiple markers according to their place. This activity builds an augmented reality based application, four 3D objects to mimic traditional music instrument which is "angklung"," kendang", "suling" and "saron", four markers used for the location of the animated 3D object, and implements virtual button on each marker. Multiple markers are designed using Vuforia software development kit. Unity $3 \mathrm{~d}$ and Vuforia SDK is used as a platform for developing the augmented reality software. The 3D objects were designed using Google sketch up 8, for imitating the Sudanese traditional music instrument such are "saron", "suling", "ending" and "angklung".

The fourth activity is integration and testing. All the units developed in the implementation phase are integrated into a system after testing of each unit. Post integration the entire system is tested for any faults and failures. Each unit is developed and tested for its functionality which is referred to as Unit Testing. The testing are done internal and externally with randomized tester with the range of age between 19 and 25 years old.

\section{RESULT AND DISCUSSION}

Fig. 1 illustrates about how software is run by the user. While running the software's, users aim at the desired marker that results in an animated augmented object apparition which they could interact. The three main component of creating a mobile augmented reality are smart phone device for the software storage, camera for scanning through the marker and a marker for a location of the animated augmented object.

Designed multiple markers using Vuforia for angklung instrument is seen in Fig. 2. A good marker will increase the chance of having an augmentable marker. The designed marker consists of two part, higher part and lower part. The higher part consists of an image illustrate an angklung object, while the lower part consists of the 8 basic musical note. Musical notes are designed to fit with the virtual button. On the right side of the marker is a result of an angklung marker generated by Vuforia SDK. As can be seen, mostly marked part are both in the area of both higher and lower part of the marker.

The designed of kendang marker is shown in Fig. 3. It consists of two part, higher part and lower part. The higher part consists of an image illustrate a "kendang" 


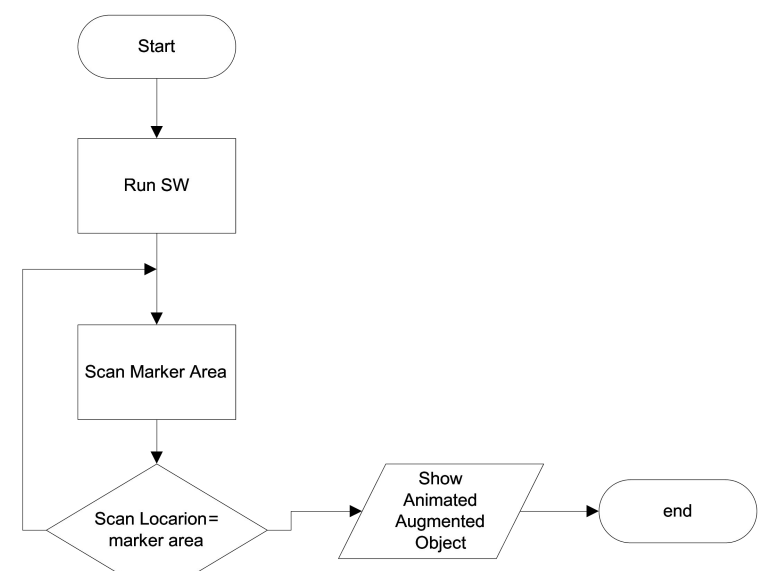

Figure 1. Augmented reality mechanism

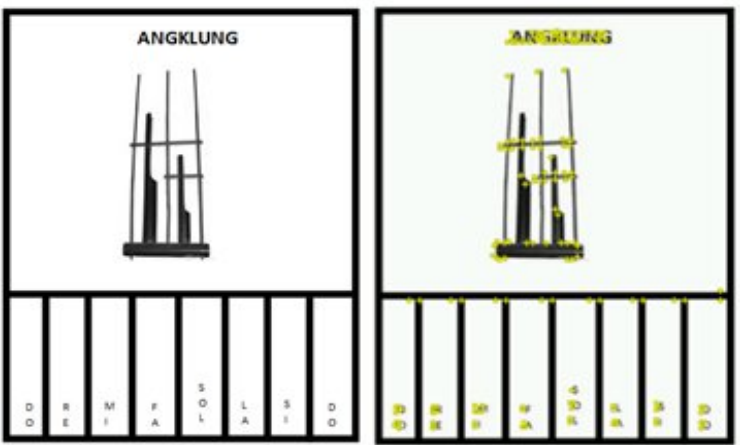

Figure 2. Angklung marker design

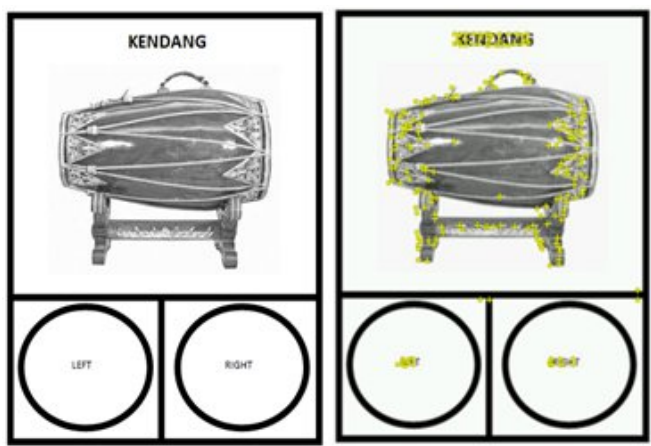

Figure 3. Kendang marker analysis

object, while the lower part consists about two circles left and right which represents the two sides of a "kendang". Musical notes are designed to fit with the virtual button. On the right side of the marker is a result of an "kendang" marker generated by Vuforia SDK. As can be seen, mostly marked part are both in the area of both higher and lower part of the marker.

Fig. 4 shows the result of all the generated markers. All markers are scored 4 from the range of 5 which means that the designed markers could be well augmentable. The 3D objects on Fig. 5, were designed using Google sketch up 8, for imitating the Sudanese

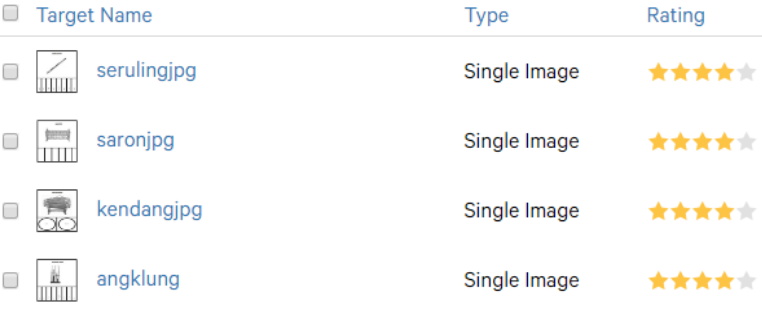

Figure 4. Augmentable marker scores

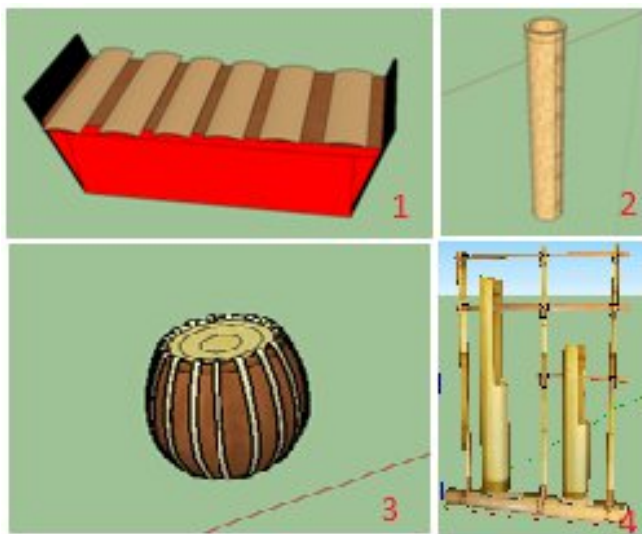

Figure 5. Traditional instruments design

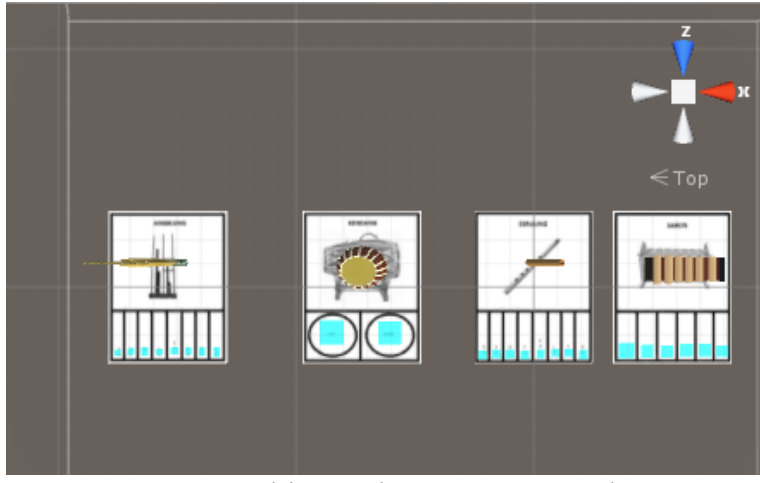

Figure 6. Objects placement on markers

traditional music instrument such are "saron","suling", "ending" and "angklung". The object will be converted to film box extension file (.fbx) for later used as an asset in the coding phase.

Fig. 6 illustrates the placement of animated 3D object on markers. Each object belongs to their related markers. The object placed is located precisely above the marker in order to get a good visualization for later use.

Fig. 7 illustrates the location of each virtual buttons on the "angklung" marker. Each virtual button is placed in adjacent to mimic piano tots. Eight amount of virtual button are needed to produce musical note sound when touched by the user. The virtual button creates an interaction experience between the user and markers. The button is not visible but the user could interact by touching area part on the marker that already marked. 


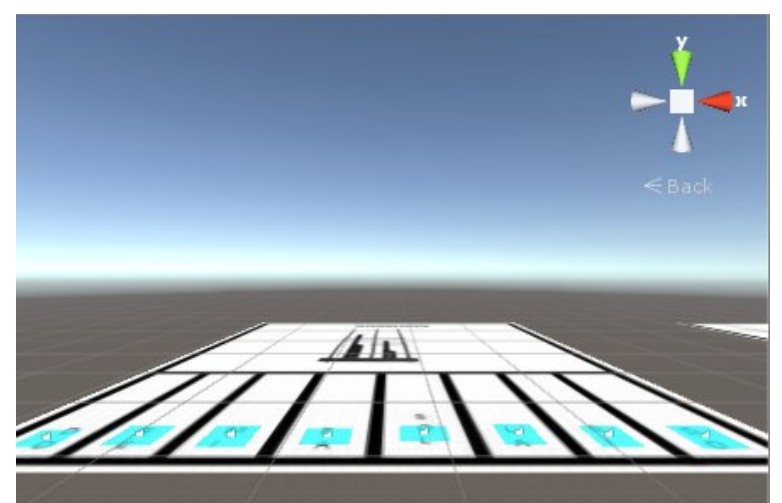

Figure 7. Virtual button on angklung marker

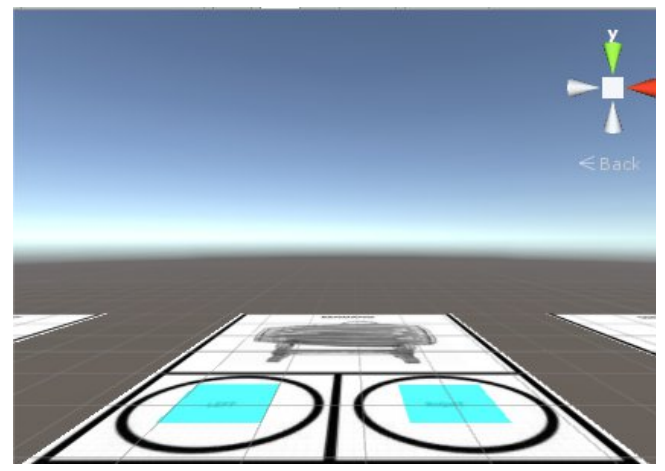

Figure 8. Virtual button on kendang marker

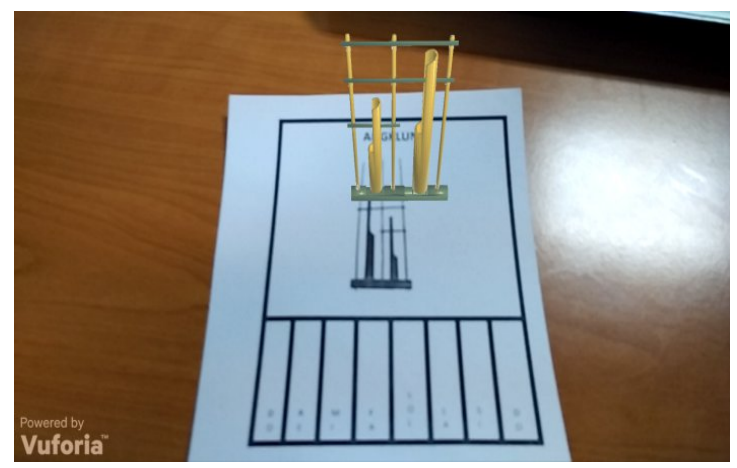

Figure 9. Angklung augmented reality

Fig. 8 illustrates the location of each virtual buttons on the "ending" marker. Two virtual buttons are used and put on the left and right side to produce the tapping sound of the "kendang" instrument.

Fig. 9 shows the output of the application above the "angklung" marker. Every time an area is scanned by a camera, the animated object will augment and the user could interact freely by touching the bottom part of the marker to hear the "angklung" traditional instrument sound.

Fig. 10 shows an output of kendang instrument visualized in augmented reality. Every time an area is scanned by a camera, the animated object will augment and the user could interact freely by touching the bottom

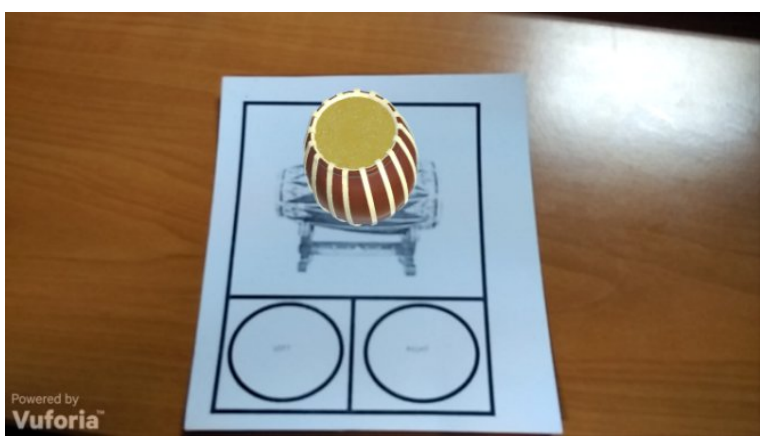

Figure 10. Kendang augmented reality

Table 1. Software testing

\begin{tabular}{cll}
\hline No & \multicolumn{1}{c}{ Test Case } & \multicolumn{1}{c}{ Result } \\
\hline 1 & $\begin{array}{l}\text { Virtual Button with marker } \\
\text { integration }\end{array}$ & Average \\
2 & 3d model appearance & High \\
3 & Augmentable markers & High \\
4 & Virtual button and audio inte- & High \\
& gration & \\
5 & Augmentable object & Average \\
6 & Speed of AR object appari- & High \\
& tion & \\
7 & Correctness of audio and vir- & High \\
& tual button & High \\
8 & Virtual Button Sensitivity & High \\
9 & Marker Scanning & High \\
10 & Application Installation & \\
& &
\end{tabular}

part of the marker to hear the tapping sound of "kendang" traditional instrument.

In this research, functional testing is conducted by observing using black box testing on the software. The test covers on the augmented object and multiple markers visualization using criteria [1], [5]. Table 1 shows result of testing cases which concluded 80 percent of the test are high and 20 percent of the test are an average so that it is suitable for preserving cultural heritage as done by [6] and [11]. The successful percentage rates gives a significant values and contribution on implementing augmented object on multiple markers instead on [3]-[5], [8] and [9] which uses single marker with augmented objects. The use of multiple markers would give benefit on user interaction experiences while using different markers at the same moment.

\section{CONCLUSION}

Based on test result, 80 percent test performed shown a high percentage, which can be considered as a successful test with minor fault. The result showed augmented reality technology is proven on imitating traditional music instruments such as sounds, visual and interaction which could serve as an alternative way to preserve digitally. 


\section{ACKNOWLEDGMENT}

We thank Universitas Trilogi, for supporting and allowing us to perform research on augmented reality related topic to further advancement on multimedia knowledge.

\section{REFERENCES}

[1] Y. Bassil, "A Simulation Model for the Waterfall Software Development Life Cycle," Int. J. Eng. Technol., vol. 2, no. 5, pp. 2049-3444, 2012.

[2] R. Yang, "The study and improvement of Augmented reality based on feature matching," in The study and improvement of Augmented reality based on feature matching, 2011, pp. 586-589.

[3] M. Figueiredo, J. Gomes, C. Gomes, and J. Lopes, "Augmented Reality Simulations for Teaching and Learning,' Int. J. Adv. Educ. Res., vol. 1, no. 1, pp. 22-34, 2014. Learning," Int. J. Adv. Educ. Res., vol. 1, no. 1, pp. 22-34, 2014.

[4] C. Hee-soo, "The Conjugation Method of Augmented Reality in Museum Exhibition," Int. J. Smart Home, vol. 8, no. 1, pp. 217-228, 2014.

[5] M. Specht, S. Ternier, and W. Greller, "Mobile Augmented Reality for Learning: A Case Study," $J$.
Res. Cent. Educ. Technol., vol. 7, no. 1, pp. 117-127, 2011.

[6] S. C. Yuen, G. Yaoyuneyong, and E. Johnson, "Augmented Reality: An Overview and Five Directions for AR in Education," J. Educ. Technol. Dev. Exch., vol. 4, no. 1, pp. 119-140, 2011.

[7] A. Mayur, K. Adwait, J. Sneha, and T. Nishi, “Augmented Reality," Int. J. Adv. Res. Comput. Sci. Manag. Stud., vol. 3, no. 2, pp. 114-122, 2015.

[8] Y. Kolstee and W. van Eck, "The Augmented Van Gogh's: Augmented Reality Experiences for Museum Visitors," in Mixed and Augmented Reality - Arts, Media, and Humanities (ISMAR-AMH), 2011 IEEE International Symposium On, 2011.

[9] Y. G. Kim and W. J. Kim, "Implementation of augmented reality system for smartphone advertisements," Int. J. Multimed. Ubiquitous Eng., vol. 9, no. 2, pp. 385-392, 2014.

[10]R. Azuma, "A survey of augmented reality," Presence Teleoperators Virtual Environ., vol. 6, no. 4, pp. 355-385, 1997.

[11] M. Antonioli, C. Blake, and K. Sparks, "Augmented Reality Application in Education," J. Technol. Stud., vol. 40, no. 2, 2014. 